NOTE

\title{
XR type-R radiochromic film $x$-ray energy response
}

\author{
Martin J Butson ${ }^{1,2}$, Tsang Cheung ${ }^{1}$ and Peter K N Yu ${ }^{1}$ \\ ${ }^{1}$ Department of Physics and Materials Science, City University of Hong Kong, Kowloon Tong, \\ Hong Kong \\ 2 Department of Medical Physics, Illawarra Cancer Care Centre, PO Box 1798, Crown Street, \\ Wollongong, NSW 2500, Australia \\ E-mail: butsonm@iahs.nsw.gov.au
}

Received 10 February 2005, in final form 18 May 2005

Published 20 July 2005

Online at stacks.iop.org/PMB/50/N195

\begin{abstract}
Gafchromic XR type- $\mathrm{R}$ radiochromic film is a relatively new product designed for use at clinical diagnostic $\mathrm{X}$-ray energies both qualitatively and quantitatively. This short note investigates the energy response characteristics of this highsensitivity radiochromic film for both diagnostic and therapeutic x-ray energies. Results are also compared to conventional silver halide $\mathrm{x}$-ray film for energy response. Results show that the energy response of the new XR type-R film is minimal over the $75-125 \mathrm{kVp}$ range (9\% variation with $\pm 3 \%$ error in measurement to $1 \mathrm{SD}$ ). This is compared to a $27 \%$ variation for $\mathrm{X}-\mathrm{Omat} \mathrm{V}$ radiographic film for the same energy range. XR type-R film does, however, produce a larger energy response variation when compared over a larger therapeutic $\mathrm{x}$-ray range $(50 \mathrm{kVp}$ superficial to $10 \mathrm{MV}$ megavoltage) with a relative response of 10.4 at $125 \mathrm{kVp}$ compared to 1 at $6 \mathrm{MV}$. This is significantly different to MD-55-2 and HS Gafchromic film which has a lower energy response at lower energies. XR type-R film is ideal for a quantitative dosimeter in the low energy range due to its relative energy independence and high sensitivity compared to conventional radiochromic film.
\end{abstract}

\section{Introduction}

Gafchromic XR type-R radiochromic film has been designed by International Specialty Products (ISP) to increase dose sensitivity at lower $\mathrm{X}$-ray energies used in diagnostic radiography as well as to produce less energy dependence in the diagnostic range. This has been performed by the addition of proprietary chemicals to vary the original film's X-ray energy and dose dependence (ISP 2004). Gafchromic XR type-R radiochromic dosimetry films have also been developed specifically for the measurement of absorbed dose of low-energy photons. Manufacturers have quoted sensitivities of these films to photons with energies less than $200 \mathrm{keV}$ as substantially greater than previous dosimetry films such as Gafchromic MD55 and HS. Due to their automatic development, radiochromic films have become significant 
dosimetry tools in diagnostic and therapeutic radiology (Vuong et al 2003, Giles 2002, Butson et al 2003). In recent times, films which are optimized for lower energy x-ray analysis (Dini et al 2003, Devic et al 2004, Chu et al 2004, Thomas et al 2004, Butson et al 2004) have been studied. For effective and accurate use in the low-energy diagnostic and therapeutic range $(75-125 \mathrm{kVp})$, a minimal energy response variation is required. This short note investigates the energy response properties of this new film and compares results to the existing radiographic films in the low energy range.

\section{Materials and methods}

Gafchromic XR type-R film is a commercially available radiochromic film product and is studied for energy dependence to X-ray radiation in this note. XR type-R film is constructed with three layers or components. The top layer consists of an approximately $100 \mu \mathrm{m}$ thick translucent yellow dye which acts as a protective barrier as well as a qualitative image enhancer, an approximately $15 \mu \mathrm{m}$ thick active layer and an approximately $100 \mu \mathrm{m}$ thick white, opaque base which acts as the image reflector for reflectance style inspection. The yellow dye aids the visual contrast of the chromatic changes that occur when the film is exposed to radiation. Gafchromic XR type-R dosimetry media not only employ the same active radiochromic components used in MD-55 film, but also include a proprietary high- $Z$ material unique to XR type-R film (ISP 2004). Gafchromic XR type-R radiochromic film (batch no J0123XRR) was used for the energy response characteristics study. For dose delivery, the films were positioned in a solid water (Constanitinou et al 1982) phantom of dimensions $30 \mathrm{~cm} \times 30 \mathrm{~cm} \times 30 \mathrm{~cm}$. At superficial and orthovoltage energies, the films were placed at the surface of the phantom for dose delivery and at megavoltage energies the films were placed at their respective $D_{\max }$ positions of $1.5 \mathrm{~cm}(6 \mathrm{MV})$ and $2.5 \mathrm{~cm}$ (10 MV). Source-to-surface distances for each type of calibrated beam were $30 \mathrm{~cm}$ for superficial beams $(50-150 \mathrm{kVp}), 50 \mathrm{~cm}$ for orthovoltage beams $(200-250 \mathrm{kVp})$ and $100 \mathrm{~cm}$ for megavoltage beams. The phantom was placed on a Pantak, Therapax 300DXT orthovoltage machine (Butson et al 1995) and a Varian 2100C linear accelerator and films were given 5 Gy absorbed dose. Absorbed dose calibrations were performed using a Farmer Thimble type ionization chamber and the IAEA TRS 277 protocol. There have recently been new protocols for calibration of megavoltage and orthovoltage x-ray beams, TRS398. This may produce variations in the beam calibration output over the entire energy range; however, this has not been taken into account with the data presented here. The effective energies of each superficial and orthovoltage beam type were calculated from half-value layer measurements. The films were irradiated while placed perpendicular to the central axis of the beam. Precautions in handling of radiochromic film outlined in TG-55 were used (Niroomand-Rad et al 1998). The film during storage and film analysis was kept at temperatures of $22 \pm 2{ }^{\circ} \mathrm{C}$, thus reducing the effects of time and temperature-dependent evolution and readout (Meigooni et al 1996) of the absorption spectra of the film. The film was only removed from a light tight envelope during irradiation and readout to reduce any effects of ambient light (Butson et al 1998). The results were measured using absorption spectra analysis using an Avantes AvaSpec-2048 reflectance photo spectrometer. The AvaSpec-2048 device is a fibre optic spectrometer with a 300 lines $\mathrm{mm}^{-1}$ grating. The bandwidth of operation is from $327 \mathrm{~nm}$ to $1100 \mathrm{~nm}$ and the instrument has a FWHM resolution of $2.4 \mathrm{~nm}$. Wavelength range of analysis was from $600 \mathrm{~nm}$ to $700 \mathrm{~nm}$ in $2 \mathrm{~nm}$ steps. This wavelength range was chosen as it forms the most sensitive region of analysis for XR type-R radiochromic film and covers the wavelength range associated with the red component of a RGB desktop scanner image. Absorption spectra data were then analysed to calculate the energy dependence of the XR type-R film. The technique 


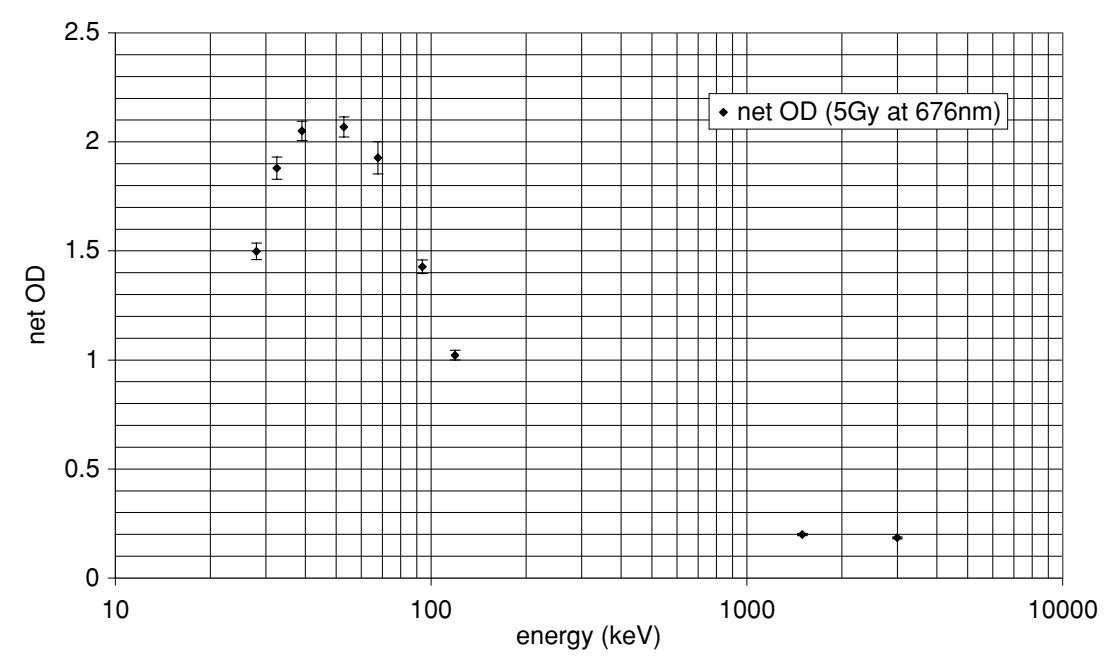

Figure 1. Energy dependence of Gafchromic XR type-R radiochromic film exposed to X-ray radiation.

for irradiation and analysis of Gafchromic HS radiochromic film and Kodak X-Omat V radiographic film are given in Cheung et al (2004) and Butson et al (2002).

\section{Results and discussion}

Figure 1 shows the energy response of the XR type-R film with 5 Gy applied dose at the $676 \mathrm{~nm}$ absorption peak wavelength of analysis. Errors shown are the measured variations produced from ten film samples at each beam energy to $1 \mathrm{SD}$. A quoted value of $\pm 3 \%$ is the average deviation over the entire data set. Effective energies for lower $\mathrm{kV}$ values are 28, 32.5, 39, 53, 68, 94 and $119 \mathrm{keV}$. The associated $\mathrm{kVp}$ values for these measured effective monoenergetic equivalents are 50, 75, 100, 125, 150, 200 and $250 \mathrm{kVp}$. Previous work using XR type-T radiochromic film showed no significant variations in energy response with wavelength readout (Cheung et al 2004). A large energy dependence is seen over the kilovoltage to megavoltage range with a peak sensitivity in response located at $53 \mathrm{keV}(125 \mathrm{kVp})$ effective energy. This value is approximately 10.4 times larger than the response for $1.5 \mathrm{MeV}$ x-rays (6 MV). Manufacturers have specified that the film is optimized for quantitative use in diagnostic and therapeutic purposes using the $\mathrm{kVp}$ range of $80-120 \mathrm{kVp}$. Our results highlight this fact with our measured sensitivities being relatively uniform (within 10\%) over the monoenergetic energy range of $32.5-68 \mathrm{keV}$ which relates to a $\mathrm{kVp}$ range of $75-150 \mathrm{kVp}$.

Figure 2 shows the energy response for Gafchromic HS film (Cheung et al 2004) and Kodak X-Omat V radiographic film with the applied doses shown in the figure at quoted wavelengths of analysis. These results show that at diagnostic energies, XR type-R film provides the highest energy independence out of the three film detectors studied. A variation of less than $9 \%$ was seen from the $\mathrm{kVp}$ range $75-150 \mathrm{kVp}(32-68 \mathrm{keV})$ for XR type-R film. The energy response variation values for Gafchromic HS and Kodak X-Omat V were approximately $17 \%$ and $27 \%$, respectively, covering the same energy range. It is also interesting to note that the energy response of XR type-R film is less than silver halide film, X-Omat V, compared to megavoltage energies as well. The maximum over-response (at $39 \mathrm{keV}$ ) compared to $6 \mathrm{MV}$ 


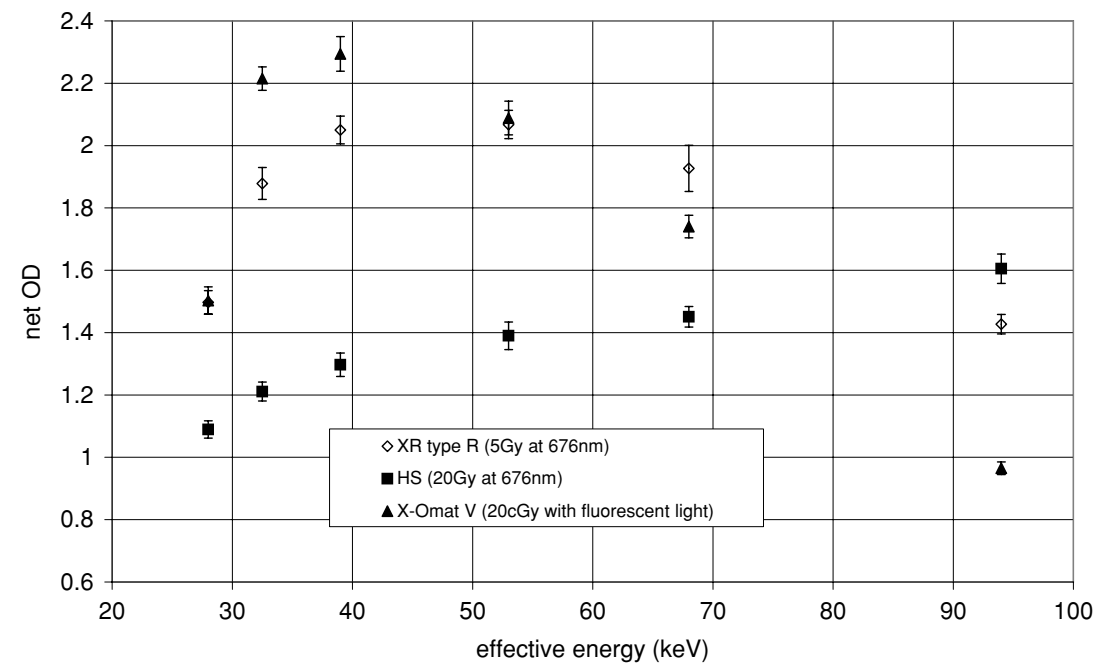

Figure 2. Comparison of energy dependence of XR type-R film to other dosimetry films used in radiotherapy and diagnostic applications.

for X-Omat was 14.5 times compared to 10.5 for XR type-R film. However, the response is also dramatically higher compared to HS which under-responses by a value of approximately 0.7 at the same energy due to its low effective atomic number. Clinically, the low energy dependence of XR type-R film makes it the most ideal film for quantitative measurement of $\mathrm{X}$-ray dose within the $75-150 \mathrm{kVp}$ energy range. It is well known that the spectral content of $\mathrm{x}$-ray beams changes with penetration into a phantom and with varying patient specific parameters; thus, a film which provides the least quantitative variation with varying energy spectrum will produce the highest level of accuracy when comparisons are made to standard condition calibration films.

\section{Conclusion}

Gafchromic XR type-R radiochromic film produces a peak energy response at $53 \mathrm{keV}$ (125 kVp) compared to other energies tested with decreasing response either side of this energy. A relatively energy-independent response (within 10\%) was found for monoenergetic equivalent beams ranging from $32.5 \mathrm{keV}$ to $68 \mathrm{keV}$ which relates to a range of $75-150 \mathrm{kVp}$. The film is significantly more sensitive to $\mathrm{x}$-rays at these kilovoltage energies with their response being approximately an order of magnitude (10.4) greater for $\mathrm{kV}$ values compared to MV. The relatively energy-independent response (with 10\%) at $\mathrm{kV}$ energies qualifies this film for consideration in quantitative analysis of dose in the $75-150 \mathrm{kVp}$-ray range associated with diagnostic procedures and superficial therapy.

\section{Acknowledgments}

This work has been fully supported by a grant from the Research Grants Council of HKSAR, China (project no CityU 100603). 


\section{References}

Butson M J, Cheung T and Yu P K 2004 Visible absorption properties of radiation exposed XR type-T radiochromic film Phys. Med. Biol. 49 N347-51

Butson M J, Mathur J and Metcalfe P E 1995 Dose characteristics of a new $300 \mathrm{kVp}$ orthovoltage machine Australas. Phys. Eng. Sci. Med. 18 133-8

Butson M J, Yu K N, Cheung T and Metcalfe P 2002 High sensitivity radiochromic film dose comparisons Phys. Med. Biol. 47 N291-5

Butson M J, Yu K N, Cheung T and Metcalfe P E 2003 Radiochromic film for medical radiation dosimetry Mater. Sci. Eng. R 41 61-120

Butson M, Yu P and Metcalfe P 1998 Effects of readout light sources and ambient light on radiochromic film Phys. Med. Biol. 43 2407-12

Cheung T, Butson M J and Yu P K 2004 Experimental energy response verification of XR type T radiochromic film Phys. Med. Biol. 49 N371-6

Chu R, Thomas G, Maqbool F, Rabe F and Burns G 2004 Measurement of dose-area product with GafChromic XR type R films Med. Phys. 31 1849-50

Constanitinou C, Attix F and Paliwal B 1982 A solid water phantom material for radiotherapy x-ray and gamma ray beam ray calculations Med. Phys. 9 436-41

Devic S, Soares C and Seuntjens J 2004 Absorption spectra of improved GafChromic film types Med. Phys. 31 1837

Dini S A, Koona R A, Ashburn J R and Meigooni A 2003 Dosimetric evaluation of type-T and type-R Gafchromic-XR film Med. Phys. 301425

Giles E R and Murphy P H 2002 Measuring skin dose with radiochromic dosimetry film in the cardiac catheterization laboratory Health Phys. 82 875-80

International Specialty Product (ISP) Incorporated (NJ, USA) 2004 Technical Data http://www.ispcorp.com

Meigooni A, Sanders M, Ibbott G and Szeglin S 1996 Dosimetric characteristics of an improved radiochromic film Med. Phys. 23 1883-8

Niroomand-Rad A, Blackwell C, Coursey B, Gall K, Galvin J, McLaughlin W, Meigooni A, Nath R, Rodgers J and Soares C 1998 Radiochromic film dosimetry: recommendation of AAPM radiation therapy task group $55 \mathrm{Med}$. Phys. 25 2093-115

Thomas G, Chu R Y and Rabe F 2003 A study of GafChromic XR type R film response with reflective-type densitometers and economical flatbed scanners J. Appl. Clin. Med. Phys. 4 307-14

Vuong T, Devic S, Belliveau P M D, Muanza T and Hegyi G 2003 Contribution of conformal therapy in the treatment of anal canal carcinoma with combined chemotherapy and radiotherapy: results of a phase II study Int. J. Radiat. Oncol. Biol. Phys. 56 823-31 